\title{
Impact of an ultrasound-driven diagnostic protocol at early intensive-care stay: a randomized-controlled trial
}

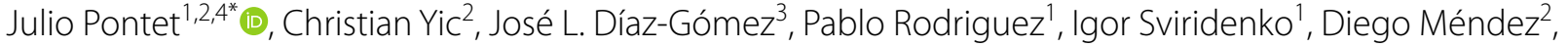
Sylvia Noveri ${ }^{1}$, Ana Soca ${ }^{1}$ and Mario Cancela ${ }^{2}$

\begin{abstract}
Background: Point-of-care ultrasound (POCUS) is a tool in increasing use, but there is still a lack of basics for its routine use and evidence of its impact in intensive care.

Objective: To measure the impact of POCUS on resource utilization, diagnostic accuracy, and clinical management in medical-surgical intensive care units (ICUs).

Methods: Prospective, controlled study, in two polyvalent ICUs. The patients were randomly assigned to POCUS or control group.

Interventions: POCUS patients received systematic ultrasound examination of optic nerve, lung/pleura, heart, abdomen, and venous system, performed at the bedside by trained intensivists. Control patients were treated by critical care specialists who do not perform ultrasound in their clinical practice.

Results: We included 80 patients, 40 per group. There were no significant differences in age, sex, APACHE II score, or admission diagnosis. POCUS group used fewer resources per patient in the first 5 days of hospitalization: chest radiography ( $2.6 \pm 2.0$ vs $4.1 \pm 3.5, P=0.01)$, additional ultrasound evaluations performed by a radiology specialist $(0.6 \pm 0.7$ vs $1.1 \pm 0.7, P=0.002)$, and computed tomography studies ( $0.5 \pm 0.6$ vs $0.9 \pm 0.7, P=0.007)$. Time to perform any requested ultrasound evaluation after ICU admission was $2.1 \pm 1.6 \mathrm{~h}$ versus $7.7 \pm 6.7 \mathrm{~h}(P=0.001)$. Systematic ultrasound evaluation led to better characterization of ICU admission diagnosis in 14 (35\%) patients and change in clinical management in 24 (60\%). POCUS group had lower fluid balance at 48 and $96 \mathrm{~h}$ after admission $(P=0.01)$ and spent less time mechanically ventilated ( $5.1 \pm 5.7$ days vs $8.8 \pm 9.4, P=0.03)$.
\end{abstract}

Conclusions: Systematic application of POCUS may decrease utilization of conventional diagnostic imaging resources and time of mechanical ventilation, and facilitate meticulous intravenous fluid administration in critically ill patients during the first week of stay in the ICU.

Trial registration ClinicalTrials.gov Identifier: NCT03608202.

Keywords: Clinical impact, Critical care, Diagnostic techniques and procedures, Point-of-care ultrasound, Point-of-care systems, Ultrasound protocol

\footnotetext{
*Correspondence: jpontet2003@gmail.com

${ }^{4}$ Larravide 2458, Montevideo, Uruguay

Full list of author information is available at the end of the article
} 


\section{Introduction}

Ultrasonography is currently considered almost essential in the management of patients in shock, acute respiratory failure, or multiorgan dysfunction [1-4]. There is still a broad field of research aimed at generating a transformational change based on scientific evidence in critical care ultrasound [5, 6]. Given the inherent limitations for an accurate physical examination of the critically ill, imaging modalities such as chest, abdominal radiography, and computed tomography (CT) are considered more reliable, useful, and impactful in the critical care setting. However, their portability, versatility, and costs are less than ideal [1], with unnecessary delays in obtaining a diagnosis in the critically ill, and increased risk for hypoxemia or refractory arterial hypotension during transportation [3]. In contrast, ultrasound, a widely available, simpler technique that can be performed with portable equipment at the bedside, has gained progressive acceptance [4].

Recent investigations have demonstrated that multiorganic ultrasound decreases resource utilization and guides clinical management of critical ill patients while enhancing diagnostic accuracy [7-11]. Nevertheless, these previous publications lack a randomized and controlled trial methodology. We hypothesize that routine use of methodic, multisystemic ultrasound protocol in critically ill patients at admission to the intensive-care unit (ICU) reduces utilization of diagnostic resources and consultation and time to definitive diagnosis and tracheal extubation. Thus, the aims of our investigation were to analyze diagnostic and therapeutic implications of a point-of-care ultrasound (POCUS)-driven protocol during the first 5 days of ICU admission.

\section{Materials and methods Study design}

Our prospective, randomized, controlled study was conducted at two major referral hospitals, Asociación Española Primera de Socorros Mutuos and Hospital Pasteur, Administración de Servicios de Salud del Estado. Patients admitted to a 12-bed medical-surgical ICU and 8-bed stepdown unit in those institutions were included in the study. The study protocol was approved by the Institutional Ethics Committee, and informed consent was obtained prior to each patient's enrollment.

\section{Patients}

We screened patients between February 1, 2017 and May 31,2017 , admitted to the ICUs. Inclusion criteria to the study were: (1) 18 years of age or older; (2) mechanical ventilation $(\mathrm{MV})$ required at ICU admission. Patients with more than $24 \mathrm{~h}$ of hospitalization and those considered with low likelihood of survival by the treating intensivist at ICU admission were excluded.

\section{Clinical data}

We collected pertinent clinical data, including Acute Physiology and Chronic Health Evaluation, second version (APACHE II) score at admission, reason for admission to the ICU, and other relevant physiologic data.

The Distensibility index of inferior vena cava (\%) was utilized as the primary measure for fluid responsiveness based on a previous investigation [12] (Table 1). In addition, we routinely used invasive MAP monitoring, capillary filling, hourly diuresis, central venous saturation, arteriovenous $\mathrm{CO}_{2}$ difference, and plasma lactate measurement alongside measure of cardiac output and LVEF as supportive parameters of the clinical-decision making of administering intravenous fluids to patients suffering from shock.

\section{Intervention}

Two groups were compared; the study group received routine evaluation with an ultrasound-driven protocol (POCUS group), while a control group received conventional management according to the pre-established protocols in the ICU (in sepsis, pneumonia, postoperative care, heart failure and myocardial ischemia, trauma, COPD, etc.).

Patients were randomly assigned by permuted blocks to either the POCUS group or the control group.

The POCUS protocol is shown in Table 1; the following variables were evaluated: confirmation of initial diagnosis; change in initial diagnosis; new unsuspected finding; lack of change in initial diagnosis, inability to rule out a condition, and no changes in treatment; association with wrong diagnosis; and association with subsequent pharmacologic, medical, procedural, or surgical measures. In addition, these resource- and outcome-related variables were analyzed for comparison between the two groups: number of additional radiologic, ultrasound, and CT studies; number and type of procedures or interventions ordered; time to for ultrasound evaluation; duration of $\mathrm{MV}$; length of stay in the ICU; and mortality rate.

POCUS studies were conducted with Logiq-e (General Electric Healthcare Japan Corporation) system with digital image storage capacity. Clinical data were saved in the SPSS Statistics 23 program (International Business Machines Corp.).

\section{Statistical analysis}

With a sample size of 40 patients per group for an annual population of 400 patients admitted to each ICU, we estimated a margin of error of $14 \%(95 \% \mathrm{CI})$. The qualitative 
Table 1 POCUS protocol

\begin{tabular}{|c|c|c|}
\hline Type of ultrasound & Instruments questions & Theoretic considerations \\
\hline Optical behavior & Diameter optic nerve: right eye (mm); left eye (mm) & $\begin{array}{l}\text { A diameter }>5.7 \text { is a noninvasive indication of intracra- } \\
\text { nial hypertension }\end{array}$ \\
\hline Neck anatomy & $\begin{array}{l}\text { Visualization of great vessels (jugular vein, carotid } \\
\text { artery): normal or abnormal }\end{array}$ & $\begin{array}{l}\text { Detection of patency (thrombosis) and anatomical } \\
\text { variants or abnormalities }\end{array}$ \\
\hline Pulmonary & Lung ultrasound score, $0-36$ points & $\begin{array}{l}\text { Score increases as pulmonary water increases; indi- } \\
\text { cates pulmonary edema }\end{array}$ \\
\hline Pleural & $\begin{array}{l}\text { Presence of pleural occupation and estimation of } \\
\text { pleural effusion }(\mathrm{mL})\end{array}$ & $\begin{array}{l}\text { Confirmation of diagnosis, volume evaluation and } \\
\text { follow-up }\end{array}$ \\
\hline Echocardiography overall function & $\begin{array}{l}\text { Estimation of left-ventricular systolic function by } \\
\text { LVEF (\%) and right-ventricular systolic function by } \\
\text { TAPSE (mm) }\end{array}$ & LVEF $>50 \%$ and TAPSE $>15 \mathrm{~mm}$ is considered normal \\
\hline Prediction of volume responsiveness & Distensibility index of inferior vena cava (\%) & $\begin{array}{l}\text { An index }>12 \% \text { indicates response to intravenous fluid } \\
\text { challenges }\end{array}$ \\
\hline Estimation of CO & $\begin{array}{l}\mathrm{CO}(\mathrm{L} / \mathrm{min}) \text { estimated by left ventricular outflow tract } \\
\text { by velocity time integral and diameter }\end{array}$ & Normal values, 4-6 L/min \\
\hline Abdominal screening & $\begin{array}{l}\text { FAST protocol for presence or absence of intraperito- } \\
\text { neal free fluid }\end{array}$ & Presence of free fluid is abnormal \\
\hline Biliary & Presence of lithiasis or dilated biliary tract & $\begin{array}{l}\text { Biliary tract pathology may be an incidental finding or } \\
\text { the cause of critical illness }\end{array}$ \\
\hline Renal & Presence of urinary lithiasis or dilated urinary tract & $\begin{array}{l}\text { Urinary tract pathology may be an incidental finding } \\
\text { or the cause of critical illness }\end{array}$ \\
\hline Ultrasound-guided invasive procedures & $\begin{array}{l}\text { Venous or arterial access, pleural or abdominal } \\
\text { drainage }\end{array}$ & $\begin{array}{l}\text { Ultrasound-guided invasive procedures are more } \\
\text { secure, with fewer adverse effects }\end{array}$ \\
\hline
\end{tabular}

CO cardiac output, FAST focused assessment with sonography in trauma, IH intracranial hypertension, LVEF left-ventricular ejection fraction, POCUS point-of-care ultrasound, TAPSE tricuspid annular plane systolic excursion

variables were records as percentages and the quantitative as mean \pm standard deviation. For the comparison of mean between groups, either the Student's $t$ test for independent groups or Mann-Whitney test was applied according to the case; and binaries in contingency tables by $x^{2}$ or Fisher's exact test. A $P$ value $<0.05$ was considered significant in all tests.

\section{Results}

\section{Patient demographics}

We included 80 patients, 40 in each group. In the POCUS group, 24 were men $(61 \%)$ and 16 were women $(39 \%)$; similarly, in the control group, there were 25 men (62\%) and 15 women $(38 \%)$. There were no statistical differences between the groups regarding age, sex, APACHE II score at $24 \mathrm{~h}$ after admission, or reason for admission to ICU (Table 2).

\section{Clinical information or decision-making following POCUS} The POCUS protocol was performed in 15-30 min in all cases.

In the POCUS group, 48 clinical decisions were made in 36 of 40 patients (90\%). Furthermore, 35\% of these observations were related to the diagnostic category, and either due to new or changed diagnosis.
Modifications in pharmacologic therapy occurred in 24 patients $(60 \%)$, and invasive maneuvers or procedures were necessary in $9(23 \%)$ (Table 3$)$.

\section{Utilization of additional diagnostic testing}

Overall, there was lower utilization of resources per patient during the first 5 days of ICU stay in the POCUS group compared with the control group (Table 4): lower number of chest radiography requested $(2.6 \pm 2.0$ vs $4.1 \pm 3.5$, respectively, $P=0.01$ ); lower utilization of additional ultrasound ICU imaging per radiology specialist $(0.6 \pm 0.7$ vs $1.1 \pm 0.7$, respectively, $P=0.002)$; and fewer $\mathrm{CT}$ scans performed $(0.5 \pm 0.6$ vs $0.9 \pm 0.7$, respectively, $P=0.007$ ).

\section{Analysis of delay times}

We measured time from admission to first ultrasound (delay time). The delay in performing diagnostic ultrasound was significantly reduced in the POCUS group versus control $(2.1 \pm 1.6 \mathrm{~h}$ vs $7.7 \pm 6.7, P=0.001)$. In the control group, all diagnostic ultrasound evaluations were performed by radiology specialists available via telephone. 
Table 2 Demographic data and diagnosis at admission in each group

\begin{tabular}{|c|c|c|c|}
\hline Groups $(N=80)$ & POCUS $(n=40)$ & Control $(n=40)$ & $P$ value \\
\hline Age, median $\pm S D, y$ & $60 \pm 15$ & $57 \pm 15$ & 0.99 \\
\hline Sex, no. (\%), male & $24(60.0)$ & $23(57.5 \%)$ & 0.99 \\
\hline $\mathrm{APACHE} \| \mathrm{I}$, median $\pm \mathrm{SD}$ & $27 \pm 9$ & $26 \pm 7$ & 0.99 \\
\hline Diagnosis at admission, no. (\%) & & & 0.99 \\
\hline Respiratory sepsis & $6(15.0)$ & $4(10.0)$ & \\
\hline Severe community-acquired pneumonia & $5(12.5)$ & $3(7.5)$ & \\
\hline Decompensated heart failure & $5(12.5)$ & $4(10.0)$ & \\
\hline Peritoneal sepsis & $4(10.0)$ & $4(10.0)$ & \\
\hline Pulmonary tuberculosis & $2(5.0)$ & $1(2.5)$ & \\
\hline Guillain-Barré syndrome & $2(5.0)$ & $1(2.5)$ & \\
\hline Sepsis unknown origin & $1(2.5)$ & $0(0.0)$ & \\
\hline Third-degree AV block & $1(2.5)$ & $2(5.0)$ & \\
\hline Stroke & $3(7.5)$ & $3(7.5)$ & \\
\hline COPD exacerbation & $2(5.0)$ & $5(12.5)$ & \\
\hline Acute myocardial infarction & $2(5.0)$ & $2(5.0)$ & \\
\hline Severe trauma & $2(5.0)$ & $3(7.5)$ & \\
\hline Suicide attempt & $2(5.0)$ & $3(7.5)$ & \\
\hline Acute bacterial meningitis & $1(2.5)$ & $0(0.0)$ & \\
\hline Pulmonary embolism & $1(2.5)$ & $1(2.5)$ & \\
\hline Thyrotoxicosis & $1(2.5)$ & $0(0.0)$ & \\
\hline Enteric sepsis & $0(0.0)$ & $2(5.0)$ & \\
\hline Acute encephalitis & $0(0.0)$ & $1(2.5)$ & \\
\hline Status epilepticus & $0(0.0)$ & $1(2.5)$ & \\
\hline
\end{tabular}

APACHE II Acute Physiology and Chronic Health Evaluation II, AV atrioventricular, COPD chronic obstructive pulmonary disease, POCUS point-of-care ultrasound

Table 3 Description of changes in clinical information or decisions led by ultrasound

\begin{tabular}{|c|c|c|}
\hline Modification in diagnosis and therapeutic decisions led by US & No. changes & No. patients \\
\hline Related to clinical decision-making, total & 48 & 36 \\
\hline $\begin{array}{l}\text { New or unidentified diagnosis: } \\
\text { Pneumonia, 2; significant pleural effusion, 5; pneumothorax, 1; significant pericardial effusion, } 1 \text {; cholecystitis, } 1\end{array}$ & 10 & 8 \\
\hline $\begin{array}{l}\text { Clinical diagnosis: } \\
\text { Pneumonia to respiratory distress due to biliary sepsis, 1; pneumonia to heart failure, 2; asthma to pneumonia, } 1\end{array}$ & 4 & 4 \\
\hline $\begin{array}{l}\text { Pharmacological therapy: } \\
\text { Fluid challenges, 6; start diuretics, 5; dobutamine, 5; noradrenaline, 2; antibiotics, 5; alteplase, } 1\end{array}$ & 24 & 16 \\
\hline $\begin{array}{l}\text { Invasive procedures: } \\
\text { Thoracic drainage, 5; emergency bronchoscopy, 2; laparotomy, 1; suprapubic bladder catheterization, } 1\end{array}$ & 9 & 7 \\
\hline Alveolar recruitment maneuver, 1 & 1 & 1 \\
\hline No changes & 0 & 4 \\
\hline
\end{tabular}

US ultrasound

\section{Clinical outcomes}

\section{Fluid management}

The POCUS-driven decision of fluid management in each patient was left at treating intensivist discretion. Similarly, clinical decisions on fluid, inotropic, or vasopressor therapy, and adjustments to MV were made based on POCUS assessment. Fluid balance (FB) was much lower in the POCUS group than in the control at 48 and $96 \mathrm{~h}$ after ICU admission $(-1600 \pm 2750 \mathrm{~mL}$ vs $-400 \pm 2130 \mathrm{~mL}, P=0.03$, and $-3200 \pm 3510 \mathrm{~mL}$ vs $-890 \pm 3900 \mathrm{~mL}, P=0.006$, respectively). There was a significant positive linear correlation between leftventricular ejection fraction (LVEF) and FB at 48 (Pearson correlation $r=0.57, P=0.002$ ) (Fig. 1) and $96 \mathrm{~h}$ $(r=0.58, P=0.03)$. 
Table 4 Comparison of resource utilization, length of stay and mortality in ultrasound-driven evaluation versus conventional management in ICU

\begin{tabular}{|c|c|c|c|}
\hline Variable & $\begin{array}{l}\text { POCUS group } \\
\text { (mean } \pm S D)\end{array}$ & $\begin{array}{l}\text { Control group } \\
\text { (mean } \pm \text { SD) }\end{array}$ & $P$ value \\
\hline Chest radiography ${ }^{\mathrm{a}}$ & $2.6 \pm 2.0$ & $4.1 \pm 3.5$ & 0.01 \\
\hline $\begin{array}{l}\text { US requested outside of } \\
\text { ICU }\end{array}$ & $0.6 \pm 0.7$ & $1.1 \pm 0.7$ & 0.002 \\
\hline Computed tomography ${ }^{a}$ & $0.5 \pm 0.6$ & $0.9 \pm 0.7$ & 0.007 \\
\hline Mechanical ventilation, days & $5.1 \pm .7$ & $8.8 \pm 9.4$ & 0.03 \\
\hline Length of stay in ICU, d & $9 \pm 8$ & $13 \pm 10$ & 0.05 \\
\hline Mortality, no. (\%) & $7(17)$ & $6(15)$ & $>0.99$ \\
\hline
\end{tabular}

Echo echocardiography, ICU intensive-care unit, US ultrasound

${ }^{a}$ During the first 5 days of intensive-care unit stay

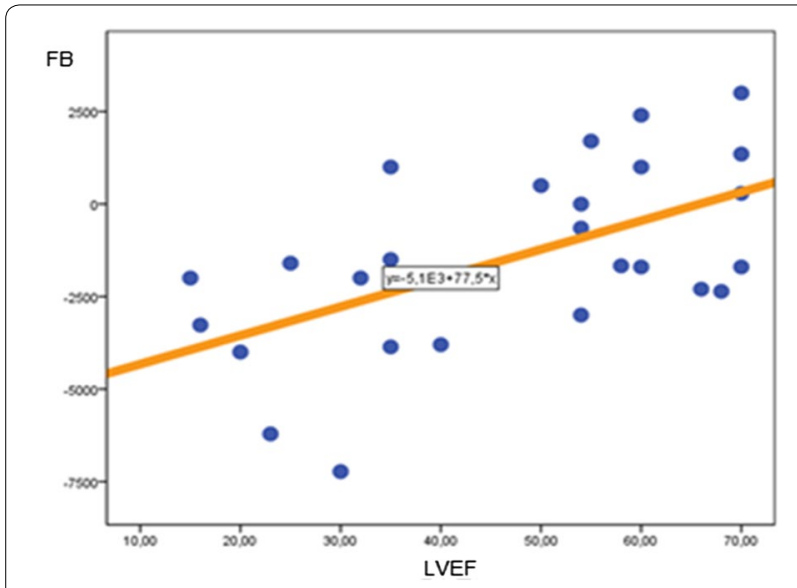

Fig. 1 Linear correlation between fluid balance at $48 \mathrm{~h}$ and left-ventricular ejection fraction. Pearson correlation $r=0.57$, $P=0.002$. FB fluid balance, LVEF left-ventricular ejection fraction

\section{Duration of $M V$}

The POCUS group showed a lower duration of MV than the control $(5.1 \pm 5.7$ days vs $8.8 \pm 9.4$ days, $P=0.03)$ (Table 4). There was no significant correlation between the time spent on MV and FB, or between the time spent on MV and LVEF.

\section{Length of stay in ICU and mortality}

Mortality rates did not differ between POCUS and control groups. Of note, there was a tendency for decreased ICU length of stay with POCUS (Table 4).

\section{Discussion}

We demonstrated the direct impact of a routine POCUSdriven protocol in patients during the early phase after admission to medical-surgical ICUs. A systematic application of POCUS resulted in decreased utilization of conventional diagnostic imaging resources and time of $\mathrm{MV}$, and facilitated a judicious intravenous fluid administration in critically ill patients during the first week of ICU hospitalization. Application of routine POCUS appears to be safe if utilized by appropriately trained intensivist as no complications were directly associated with POCUS use in the study group.

Previous investigations $[2,5]$ applying particular ICUsound protocol led to more accurate admission diagnosis in $25.6 \%$ to $24.9 \%$. They had an observational design, but are comparable to the $22 \%$ accuracy rate of new or corrected diagnoses in our study. Moreover, the majority of our patient population suffered from sepsis. Manno et al. [2] proposed that septic patients may be the most favored subgroup of patients receiving a POCUS protocol because of the myriad of information and therapies that can be tailored to each individual patient.

The main effect of routine utilization of our POCUS protocol was the clinical decision-making, more specifically in pharmacologic management adjustments in shock patients $(60 \%)$ and ordering of ultrasound-guided procedures (23\%). In our study, we found that $23 \%$ of our population required ultrasound-guided invasive procedures, which is similar to the $21.6 \%$ reported by the Manno group [2]. Vignon et al. [13] described a change in therapeutic management of patients in $51 \%$ of the cases, and Bernier-Jean et al. [5] in 44\%.

Management of intravascular volume status can be a difficult decision at the bedside. Meticulous fluid management in treating shock patients with heart disease is desirable. In our case, the most common clinical decision was related to fluid management. Similar findings have been described in an observational study [13], but we emphasize the randomized-controlled design of our study. Nonetheless, our direct comparison of timely ultrasound-driven versus conventional management allows us to represent the current practice in the majority of medical centers due to lack of routine utilization of ultrasound by intensivists. Furthermore, positive fluid responsiveness test does not indicated necessity of further fluid administration, our clinical-decision making was determined by the echocardiography measurements (LVEF, IVC distensibility index) in addition to systemic perfusion surrogates (central venous saturation, arteriovenous $\mathrm{CO} 2$ difference, and plasma lactate measurements). Finally, in cases of heart failure, we guided restriction of fluids by signs of pulmonary and systemic congestion. However, we acknowledge tolerance to fluids must be assessed with diastolic function parameters and may be in addition to lung ultrasonography [14-16].

Our POCUS protocol led to a significantly lower utilization of chest radiography, ultrasound performed by nonintensivist specialists, and CT scans (up to $56 \%$ reduction in $\mathrm{CT}$ requests). We found a reduction of radiology 
evaluation utilization from 4.1 in the control group to 2.6 in the POCUS group (63\% reduction). This finding differs from other groups reporting $22 \%$ to $26 \%$ reduction in radiography utilization $[17,18]$. Possible explanations include intensivist discretion versus specific protocol, considerable critical care ultrasound expertise by attending intensivist at time of the study, and more availability of fully capable ultrasound devices. Of note, the American College of Radiology expert panel recommended daily radiology for each intubated patient [17]. The direct clinical implications of our findings were associated with lower radiation exposure, less intra-hospital transportation of unstable patients, and inherent economic savings with more sophisticated evaluations, including in improved imaging suites workflow and reduced necessity for personnel to transport patients [19-21].

We found a significant correlation between cardiac function (left systolic ventricle function measured by LVEF index) and a negative FB in the first 5 days of ICU management. This might indicate that judicious fluid management and decisions in administration of diuretic agents are facilitated with real-time utilization of POCUS since ICU admission. We did not find a significant correlation between time spent on MV and FB or between duration of MV and LVEF. This can possibly be explained by the heterogeneity of our population. However, there was correlation between lower LVEF and negative FB, since patients with low LVEF are likely to receive less fluids and more diuretics.

We acknowledge several limitations in the current investigation. First, our small patient population avoids finding significant differences in meaningful clinical outcomes, such as mortality. Second, we analyzed only the first 5 days of ICU stay. However, as patients stay longer in the ICU, additional confounding factors not necessarily related with admission diagnosis can affect the outcomes (late-onset, ventilator-associated pneumonia). Mortality was similar between both groups, but it should be noted that future studies with larger patient populations having a shorter time of MV as found in the POCUS group could potentially determine a decrease in mortality related to MV complications. Third, we did not find a significant correlation between normal or high ejection fraction and pulmonary $B$ profile in our study that can imply possible tolerance to fluid administration. However, patients with $\mathrm{CHF}$ and preserved EF can be only characterized with advanced echocardiography parameters of diastolic function. We recognize that the lack of diastolic function assessment is another limitation of this study. However, we aimed to evaluate a practical approach for assessment of ventricular function and fluid responsiveness, so we propose further investigation of the correlation between normal or high ejection fraction and pulmonary B profile in addition to diastolic function assessment in septic shock patients.
Finally, the absence of a reposition protocol guided by pre-established ultrasound should be endorsed in multicentric studies.

\section{Conclusions}

A systematically applied POCUS protocol upon admission to the ICU can guide diagnostic and therapeutic decisions in critically ill patients. This potentially leads to greater resourcefulness and shorter duration of MV. Definitive associations, such as ultrasound-driven fluid resuscitation and improved outcomes, remain to be proven in larger multicenter studies. Diastolic function assessment can improve overall evaluation of fluid tolerance to the fluid management of septic shock patients.

\section{Acknowledgements \\ Stella Calvo, MD for his technical help in the elaboration of the manuscript. Preliminary results and abstract presented at 13th WINFOCUS World Congress, Argentina, September 2017, and received the Golden Probe Award as Best Poster Presentation.}

\section{Authors' contributions}

JP and CY have made substantial contributions to the conception and design of the work, the acquisition, analysis, and interpretation of data, and have drafted the work. JLD have made contributions to the analysis and interpretation of data and substantively revised it. PR, IS, and DM have made contributions to the acquisition and analysis of data. SN, AS, and MC have made contributions to the analysis and interpretation of data and substantively revised it. All authors read and approved the final manuscript.

\section{Funding}

There was no source of funding.

\section{Availability of data and materials}

The data sets used and/or analysed during the current study are available from the corresponding author on reasonable request.

\section{Ethics approval and consent to participate}

The study protocol was approved by the Institutional Ethics Committee (Hospital Pasteur), and informed consent was obtained prior to each patient's enrollment.

\section{Consent for publication \\ Not applicable.}

\section{Competing interests}

The authors declare that they have no competing interests.

\section{Author details}

${ }^{1}$ Intensive Care Unit, Hospital Pasteur, Administración de Servicios de Salud del Estado, Montevideo, Uruguay. ${ }^{2}$ Intensive Care Unit, Asociación Española Primera de Socorros Mutuos, Montevideo, Uruguay. ${ }^{3}$ Department of Critical Care Medicine, Mayo Clinic, Jacksonville, USA. ${ }^{4}$ Larravide 2458, Montevideo, Uruguay.

Received: 7 June 2019 Accepted: 20 September 2019

Published online: 30 September 2019

\section{References}

1. Frankel HL, Kirkpatrick AW, Elbarbary M et al (2015) Guidelines for the appropriate use of bedside general and cardiac ultrasonography in the evaluation of critically ill patients—part I: general ultrasonography. Crit Care Med 43:2479-2502 
2. Manno E, Navarra M, Faccio L et al (2012) Deep impact of ultrasound in the intensive care unit: the "ICU-sound" protocol. Anesthesiology 117:801-809

3. Moore CL, Copel JA (2011) Point-of-care ultrasonography. N Engl J Med 364:749-757

4. Narasimhan M, Koenig SJ, Mayo PH (2016) A whole-body approach to point of care ultrasound. Chest 150:772-776

5. Bernier-Jean A, Albert M, Shiloh AL, Eisen LA, Williamson D, Beaulieu $Y$ (2017) The diagnostic and therapeutic impact of point-of-care ultrasonography in the intensive care unit. J Intensive Care Med. 32:197-203

6. Bossone E, DiGiovine B, Watts S et al (2002) Range and prevalence of cardiac abnormalities in patients hospitalized in a medical ICU. Chest 122:1370-1376

7. Kristensen MS, Teoh WH, Graumann O, Laursen CB (2014) Ultrasonography for clinical decision-making and intervention in airway management: from the mouth to the lungs and pleurae. Insights Imaging. 5:253-279

8. Laursen CB, Sloth E, Lassen AT et al (2014) Point-of-care ultrasonography in patients admitted with respiratory symptoms: a single-blind, randomised controlled trial. Lancet Respir Med. 2:638-646

9. Oks M, Cleven KL, Cardenas-Garcia J et al (2014) The effect of point-ofcare ultrasonography on imaging studies in the medical ICU: a comparative study. Chest 146:1574-1577

10. Pontet J, Yic C, Diaz-Gomez J, et al. Ultrasonografía En Medicina de Urgencias, Terapia Intensiva Y Anestesia. 1st ed: Montevideo; 2016

11. Volpicelli G, Lamorte A, Tullio M et al (2013) Point-of-care multiorgan ultrasonography for the evaluation of undifferentiated hypotension in the emergency department. Intensive Care Med 39:1290-1298

12. Feissel M, Michard F, Faller JP, Teboul JL (2004) The respiratory variation in inferior cava diameter as a guide to fluid therapy. Intensive Care Med 30(9):1834-1837

13. Vignon P, Frank MB, Lesage J et al (2004) Hand-held echocardiography with Doppler capability for the assessment of critically-ill patients: is it reliable? Intensive Care Med 30:718-723
14. Geri G, Vignon P, Aubry A, Fedou AL, Charron C, Silva S, Repessé X, Vieillard-Baron A (2019) Cardiovascular clusters in septic shock combining clinical and echocardiographic parameters: a post hoc analysis. Intensive Care Med 45(5):657-667

15. Nair S, Sauthoff H (2019) Assessing extravascular lung water with ultrasound: a tool to individualize fluid management? J Intensive Care Med. https://doi.org/10.1177/0885066619855000 (Epub ahead of print)

16. Orme RM, Oram MP, McKinstry CE (2009) Impact of echocardiography on patient management in the intensive care unit: an audit of district general hospital practice. Br J Anaesth 102:340-344

17. Expert Panel on Thoracic Imaging, McComb BL, Chung JH et al (2016) ACR appropriateness criteria routine chest radiography. J Thorac Imaging. 31:W13-15

18. Krinsley JS (2003) Test-ordering strategy in the intensive care unit. J Intensive Care Med. 18:330-339

19. Expert Round Table on Echocardiography in ICU (2014) International consensus statement on training standards for advanced critical care echocardiography. Intensive Care Med 40:654-666

20. Kue R, Brown P, Ness C, Scheulen J (2011) Adverse clinical events during intrahospital transport by a specialized team: a preliminary report. Am J Crit Care. 20:153-161 (quiz 162)

21. Smith I, Fleming S, Cernaianu A (1990) Mishaps during transport from the intensive care unit. Crit Care Med 18:278-281

\section{Publisher's Note}

Springer Nature remains neutral with regard to jurisdictional claims in published maps and institutional affiliations.

\section{Submit your manuscript to a SpringerOpen ${ }^{\circ}$ journal and benefit from:}

- Convenient online submission

- Rigorous peer review

- Open access: articles freely available online

- High visibility within the field

- Retaining the copyright to your article

Submit your next manuscript at $\boldsymbol{\nabla}$ springeropen.com 